\title{
Research on Computer Integrated Manufacturing of Sheet Metal Parts for Lithium Battery
}

\author{
Wei-Min PAN1 ${ }^{1, a}$, Guo-Hua LI ${ }^{1, b}$ and Meng-Han LI ${ }^{1}$ \\ ${ }^{1}$ College of Mechanical and electrical engineering, Henan University of Science \& Technology, Henan province, China \\ Email: ${ }^{2}$ Wmpool1@163.com, ${ }^{b}$ liguohua311@163.com
}

\begin{abstract}
Lithium battery has been widely used as the main driving force of the new energy vehicle in recent years. Sheet metal parts are formed by means of pressure forming techniques with the characteristics of light weight, small size and high structural strength. The sheet metal forming has higher productivity and material utilization than the mechanical cutting, therefore sheet metal parts are widely used in many fields, such as modern automotive industry, aviation, aerospace, machine tools, instruments and household appliances. In this paper, taking a complex lithium battery box as an example, the integrated manufacturing of sheet metal parts is studied, and the digital integrated design and manufacturing process system is proposed. The technology is studied such as sheet metal design, unfolding, sheet nesting and laser cutting, CNC turret punch stamping programming, CNC bending etc. The feasibility of the method is verified through the examples of products and the integrated manufacturing of sheet metal box is completed.
\end{abstract}

\section{Introduction}

With the rapid development of new energy vehicles and electric vehicles, lithium battery has been widely used as the main driving force in recent years. Sheet metal parts are formed by means of pressure forming techniques with the characteristics of light weight, small size and high structural strength. The sheet metal forming has higher productivity and material utilization than the mechanical cutting, therefore sheet metal parts are widely used in many fields, such as modern automotive industry, aviation, aerospace, machine tools, instruments and household appliances. Nowadays the digital integrated manufacturing technology and the computer-aided engineering software have been used to achieve the integration of design and manufacturing in the production of sheet metal parts [1].

In this paper, taking a complex lithium battery box as an example, using UG, Bysoft, and AP100 software, the integrated manufacturing of sheet metal parts is studied, and the digital integrated design and manufacturing process system is proposed. The technology is studied such as sheet metal design, unfolding, sheet nesting and laser cutting, $\mathrm{CNC}$ turret punch stamping programming, CNC bending etc. The feasibility of the method is verified through the examples of products.

\section{Digital Integrated Manufacturing Process}

In modern sheet metal industry, the computer aided design and manufacturing technology have been widely used. The computer-aided design software is used for $2 \mathrm{D}$ and $3 \mathrm{D}$ design of sheet metal parts. The sheet is machined by punching, molding and other processing methods. The product is finished through weld, renovation, painting and assembly [2].

In the manufacturing process, the computer-aided manufacturing software is utilized for unfolding, nesting, generating $\mathrm{NC}$ programs etc. The digital integrated manufacturing process is shown in Figure 1.

\section{Sheet Metal Design}

With the industrial development, the parametric design and CAD integration technology based on the constraint are paid more and more attention. The so-called parameterization is that the design requirement, principle, method and result are indicated with flexible parameters, the parameter can be changed based on the actual situation in the course of human-computer interaction. The CAD system is that the constraint is used to express the shape characteristics of the product model, the parameters are defined to control the design result, thus the design model can be modified by adjusting the 
parameters, and a series of design programs can be easily created similar in shape or feature [3].

In sheet metal design industry, the modelling method and sculpting method are main design method by using CAD. The sculpting method is the most widely used and CAD. The sculpting method is the most widely used and the model method is a traditional method of sheet metal design. The regular surface modeling is used for sheet metal parts with simple structure shape sheet. The surface modeling and solid modeling are used for sheet metal parts with complex structure such as scanning, pulling, spiral, cutting and other functions. In this paper, the sheet metal part is a lithium battery box, and the threedimensional modeling is completed by using the sheet metal module of UG. The model is shown in Figure 2.

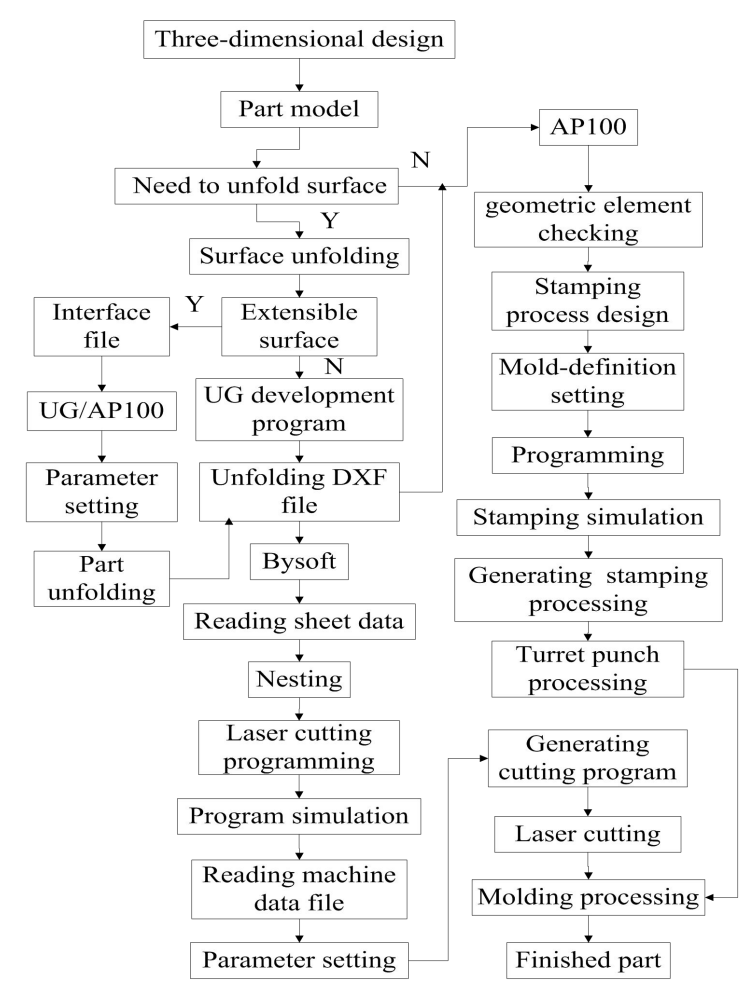

Figure 1. Digital integrated design and manufacturing process.

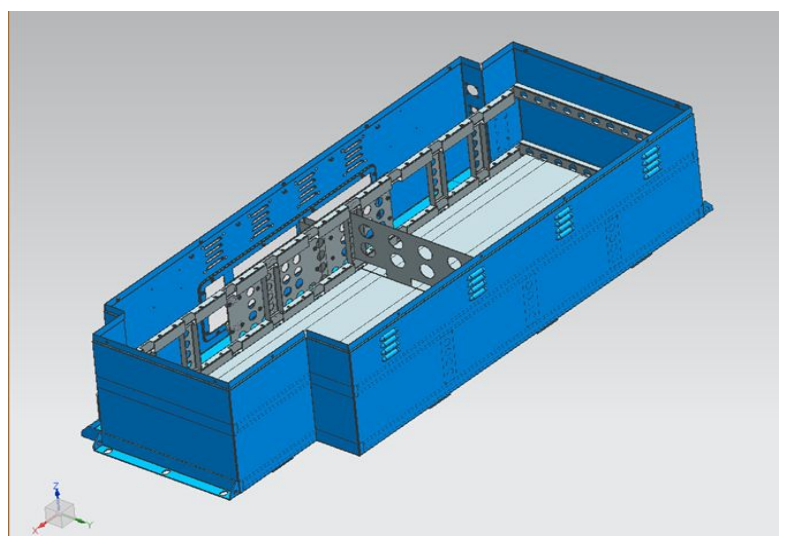

Figure 2. Box model.

\section{Sheet Metal Unfolding}

Compared with the general machinery part, the sheet metal part has its own characteristics in the structure, material and technology. In modern sheet metal processing, it is very important to design reasonably the basic parameter and manufacturing, and draw accurately the unfolding view [4].

The length of the neutral layer is usually considered as the developed length of sheet metal parts for the developable surface. The unfolded size of the plane is shown in Figure 3.

$$
L=\frac{\pi \times(R+K T) \times \theta}{180}
$$

Where, $L$ is the developed length; $R$ is the inner bending radius; $\theta$ is the bending angle; $T$ is the thickness of the material; $K$ is the factor of neutral layer, $K=t / T$; $t$ is the distance between the inner surface and the neutral plane. It can be seen from Eq. 1 that the developed length is determined mainly by the bend angle $\theta$, the neutral layer coefficient $K$, the material thickness $T$. Notches or regional block processing are usually carried out at first in unfolding the surface with complex structure or the closed surface. The unfolding of the complex surface is transformed into the unfolding of one or a few relatively simple surfaces.

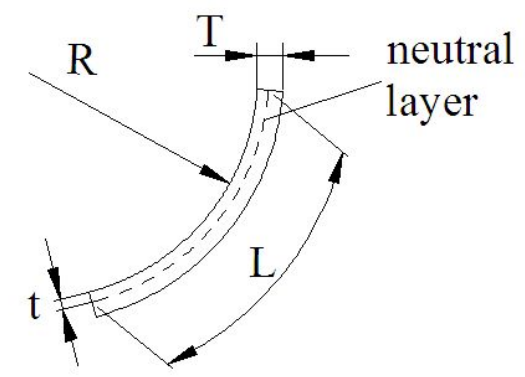

Figure 3. Expanded dimension calculation.

The approximate algorithm is used to unfold the nondevelopable surface. The surface is divided into many small quadrilateral mesh elements before the unfolding and the mesh elements are converted into the same plane. The developable size is got by adopting approximately numerical methods of equal area and developable algorithms of quadrilateral mesh elements. The file of the unfolded surface is obtained by reconstructing the model at last.

Part $\mathrm{a}$ and part $\mathrm{b}$ are selected from the box. The threedimensional graphics and the developable view of part a and part $b$ are shown in Figure 4 and Figure 5, they are unfolded by using the sheet metal module of UG or AP100 software. The maximum developable size of part a is $180.3 \mathrm{~mm} \times 105.3 \mathrm{~mm}$, and the thickness is $1.5 \mathrm{~mm}$; the maximum developable size of part $b$ is $570.1 \mathrm{~mm} \times$ $65.3 \mathrm{~mm}$, and the thickness is $1.5 \mathrm{~mm}$. 

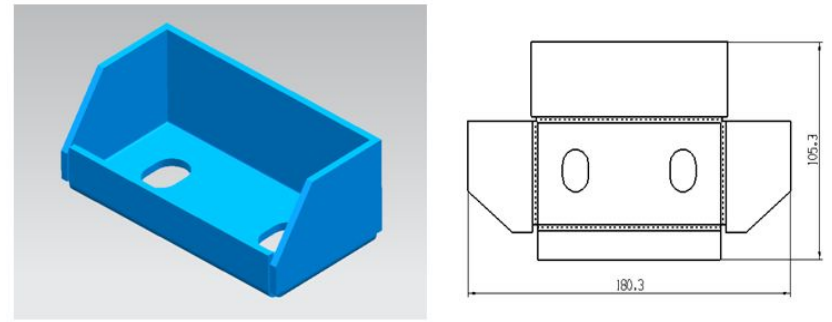

Figure 4. Three-dimensional and unfolded graphics of part a.

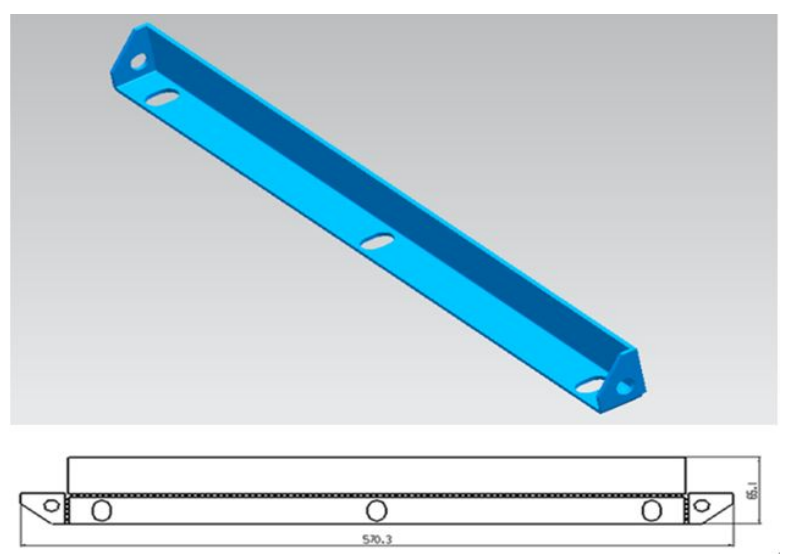

Figure 5. Three-dimensional and unfolded graphics of part $b$.

\section{Sheet Nesting and Cutting}

The more variety and less bulk are often required in the sheet metal production. In order to shorten the production cycle and improve the material utilization, the parts with the same material and thickness are generally arranged on the same sheet to machine in one clamping, therefore the problem of optimizing nesting must be solved. The nesting is generally divided into the non-constrained nesting and the constrained nesting. In terms of optimizing nesting, a computer is stronger than manual nesting. The nesting result is rapidly given by the sheet metal software according to the processing condition and the machine parameter in nesting. The user can make some changes on the automatic nesting to meet special production requirement [5].

In modern industrial manufacturing field, the cutting is one basic process with a large amount of application. The modern cutting technology is on the basis of modern computer technology. For different cutting equipments, the traditional cutting technology is improved to develop into the computer-aided cutting production and management software with the technology of nesting optimization, sheet metal unfolding and remaining material database management as the core. The software can be used to improve the comprehensive utilization of the steel and the cutting efficiency and cutting quality [6].

The Swiss Bystronic laser cutting machine is used. The Bysoft software is utilized for nesting and cutting to make full use of the material by using mixed nesting methods with the constraint. The overall dimension of the sheet is $2500 \mathrm{~mm} \times 1250 \mathrm{~mm}$, and the thickness is 1.5 $\mathrm{mm}$. Part a and part $\mathrm{b}$ will be cut on a plate, and the number of part a is 12 , and the number of part $\mathrm{b}$ is 8 . The angle is not limited in material fiber direction in the cutting, and the distance between the two parts is set independently. The automatical nesting result is shown in Figure 6.

\section{CNC punching and bending}

CNC punching techniques can be used to automatically punch the shape on the sheet, reduce die wear with a standard mold and get high production efficiency. It is necessary to accurately calculate the punching pressure when punching the plate with a greater thickness and a relatively large hole.

$$
F=C \times T \times K \times \tau \times 0.345
$$

Where, $F$ is the press force, and the unit is $k N ; C$ is the circumference of the punched hole, and the unit is $\mathrm{mm} ; T$ is the thickness of the material, and the unit is $\mathrm{mm}$; $K$ is the factor of the material; $\tau$ is the shear factor.

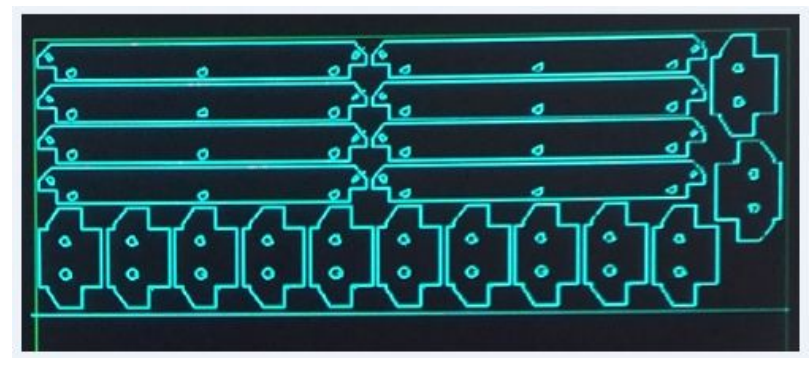

Figure 6. Nesting result.

For the large-diameter round or square holes, if $F$ is greater than the punching pressure of the CNC turret punch, the holes can be completed in many stamping. The principle of procedure concentration should be followed in the stamping, namely the all processible holes and the profile of the part are possibly completed in one clamping to prevent positioning errors from repeating. If the positioning must be repeated, the hole with the position tolerance is completed in one stamping. The molds should be choose with a reasonable order to stamping according to some rules, and the rules are that the round hole is before the square hole, the small hole is before the big hole, the middle before the profile. When designing the stamping process, the rigidity of the work is improved by adding the micro connection between two parts on one sheet to avoid the interference between the part warpage and the movement of the turret and the die [7].

The CNC punching procedure is programmed with AP100 software. The analog processing method is used to check the reliability of the CNC program. In this paper, part a is unfolded to punch with the CNC turret punch and programmed with AP100 software. A part of CNC punching program of part a is shown in figure 7. 


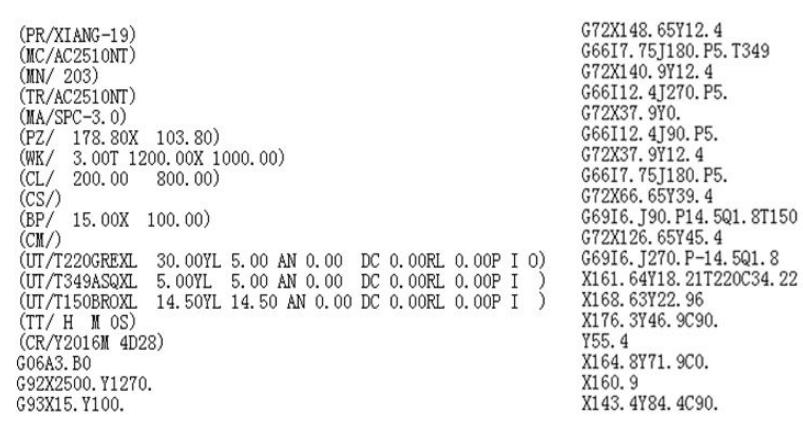

Figure 7. CNC punching Programming.

The bending method is used most commonly to achieve a specific sheet metal structure. The CNC bending machine can be used to obtain specific sheet metal forming process and realize the bending process precisely and rapidly. The finished box is shown in Figure 8 . The box has a relatively regular geometric structure. The internal ribs are used for strengthening the structure; the shutters in both sides are used for cooling; the external welding parts are conducive to moving and fixing. The box body is mainly used in the electric vehicle to install the lithium battery.

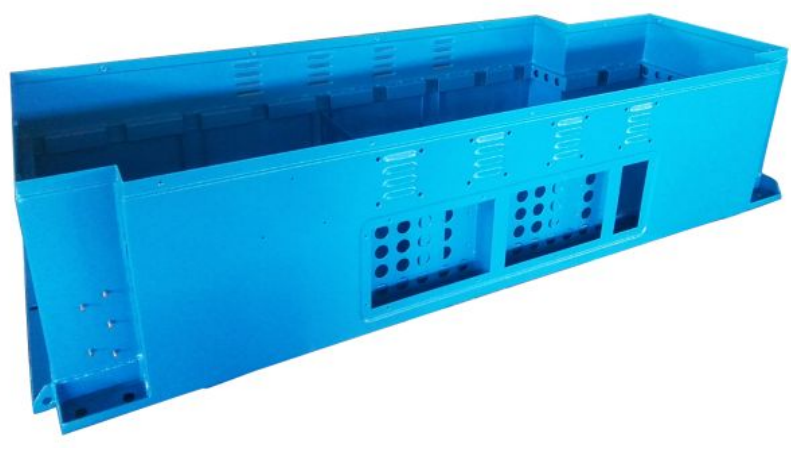

Figure 8. Finished product.

\section{Conclusion}

The CAD / CAM technology is used in the sheet metal design and processing. Based on the three-dimensional modeling of sheet metal parts of the lithium battery box, the data in unfolding, nesting, laser cutting, CNC punching and other aspects are shared and the integrated manufacturing is completed. The strong technical support is provided to accelerate the product development, improve the product quality and enhance the market competitiveness for enterprises.

\section{References}

1. J.S. Huang, A DFM Oriented Sheet Metal CAD and Its Development (Southeast University, China 2008)

2. J.Z. Xia, Practitioners of Sheet Metal (Mechanical Industry Press, China 2001)

3. X.X. Meng, Y.N. Xu, A Survey of the Research Works on Parametric Design, Journal of Computeraided Design \& Computer Graphics, 14, 11 (2002)
4. J.Y. Liu, Research on Key Techniques of Unfolding and Optional Nesting System of Sheet Metal Parts (Xi'an Technological University, China 2011)

5. Z.X. Jia, State-of-the-art and Future Trends of Cutting and Packing Studies, Journal of Computeraided Design \& Computer Graphics, 16, 7 (2004)

6. Y.W. Zhu, Research on modern incision technology and its application in enterprise, Technological Development of Enterprise, 24, 8 (2005)

7. M.F. Wu, H. Jin, M. Chen, Technology Research for Numerical Control Machine of Rotate Punch Tower, Electro-Mechanical Engineering, 26, 1 (2010) 